\title{
Noise induced calcium oscillations in a cell exposed to electromagnetic fields ${ }^{1}$
}

\author{
Yuhong Zhang ${ }^{\mathrm{a}, \mathrm{b}}$, Yongli Zhao ${ }^{\mathrm{c}}$, Yafei Chen ${ }^{\mathrm{a}, \mathrm{b}}$, Changqing Yuan ${ }^{\mathrm{a}, \mathrm{b}}$ and Yong Zhan ${ }^{\mathrm{a}, \mathrm{b},{ }^{*}}$ \\ ${ }^{a}$ Hebei Province Key Laboratory on Molecular Biology, Tianjin 300401, China \\ ${ }^{b}$ School of Sciences, Hebei University of Technology, Tianjin 300401, China \\ ${ }^{c}$ Advanced Mechatronics Equipment Technology, Tianjin Major Laboratory, Tianjin 300387, China
}

\begin{abstract}
The effects of noise on the calcium oscillations in a cell exposed to electromagnetic fields are described by a dynamic model. Noise is a very important factor to be considered in the dynamic research on the calcium oscillations in a cell exposed to electromagnetic fields. Some meaningful results have been obtained here based on the discussion. The results show that the pattern of intracellular calcium oscillations exposure to electromagnetic fields can be influenced by noise. Furthermore, the intracellular calcium oscillations exposure to electromagnetic fields can also be induced by noise. And the work has also studied the relationships between the voltage sensitive calcium channel's open probability and electromagnetic field. The result can provide new insights into constructive roles and potential applications of selecting appropriate electromagnetic field frequency during the research of biological effect of electromagnetic field.
\end{abstract}

Keywords: Calcium oscillations, noise, electromagnetic fields, open probability

\section{Introduction}

It is well known that intracellular calcium oscillations play a significant role in the process of cell regulation, e. g., calcium oscillations can increase the efficiency and specificity of gene expression, and regulate cellular process from egg fertilization to cell death, etc. $[1,2]$. Intracellular calcium concentration $\left(\left[\mathrm{Ca}^{2+}\right]_{\mathrm{i}}\right)$ exposure to electromagnetic fields was measured by many researchers [3-6], and the biological effects of electromagnetic fields should be noted. A number of theoretical models have been proposed to explain the oscillatory behavior of calcium ions in a cell exposed to electromagnetic fields [7-11]. For example, Huang et al. [8] reported the influence of electromagnetic fields on intracellular calcium concentration through the experiment research of osteoblasts [8]. Zhang et al. found that the frequency factor and the energy factor results at the window effects [9]. Another work has shown that cytosol calcium oscillation could be induced by some types of electromagnetic fields [10].

Noise plays a constructive role in many nonlinear systems and its effect has been widely studied in the past two decades. As a result of the number of membrane receptors, ion channels, and calcium ions

\footnotetext{
${ }^{1}$ All authors disclose no conflicts of interest.

* Address for correspondence: Yong Zhan, Hebei Province Key Laboratory on Molecular biology, Hebei University of Technology, Tianjin 300401, China. Tel.: +86-22-60435638; Fax: +86-22-83955258; E-mail: zhany@hebut.edu.cn.
} 
in some organelles is very low [12], noise effects cannot be neglected. Now that noise is an important factor to be considered, its effects on the process of dynamic research should be taken into consideration further. Are there effects of noise on the calcium oscillations in a cell exposed to electromagnetic fields? This is a starting point from which the work would analyze in the following discussion.

In this paper, the Goldbeter model [13] is applied to get insight into the effects of noise on the behavior of calcium oscillations in a cell exposed to electromagnetic fields. The results show that the pattern of cytosol calcium oscillations exposure to electromagnetic fields can be influenced as well as induced by noise.

\section{The model}

Following the previous work [10], the effects of noise on the intracellular calcium oscillations exposure to electromagnetic fields will be discussed. For the dynamic model, $X$ is cytosolic calcium concentration and $Y$ is calcium concentration in the $\operatorname{InsP}_{3}$-insensitive pool. And the dynamic model is described by the following set of equations:

$$
\begin{gathered}
\frac{d X}{d t}=v_{0} \times \frac{P_{0}}{P_{0}^{\prime}}-v_{1}+v_{2}+k_{f} Y-k X+\sqrt{2 D} \xi(\mathrm{t}) \\
\frac{d Y}{d t}=v_{1}-v_{2}-k_{f} Y
\end{gathered}
$$

Eqs. (1) and (2) were originally proposed by Goldbeter [13] to explain calcium oscillations in a cell. Detailed description of other parameters in this model can be found in reference [13], and the parameter values in Eqs. (1) and (2) are chosen according to reference [13]. The number of reaction molecules is often low in a cell [14]. Then noise resulted from random fluctuations should be considered in this model. $D$ denotes the intensity of Gaussian white noise $\xi(t)$ with zero mean value $<\xi(t)>=0$ and unit variance $\left\langle\xi(t) \xi\left(t^{\prime}\right)\right\rangle=\delta\left(t-t^{\prime}\right)$.

The experiment of Grassi, et al. [15] showed that the $\mathrm{Ca}^{2+}$ current through a calcium channel is almost unaffected by electromagnetic fields. And the enhancement of $\mathrm{Ca}^{2+}$ current density was due to the increased number of voltage-gated $\mathrm{Ca}^{2+}$ channels. Ebrahimian [16] had also drawn a conclusion that low frequency electromagnetic fields with $50 \mathrm{~Hz}$ frequency will directly lead to change in bioelectric activities neurons through a change in amount and rate of open and close ion channels. In conclusion, the exposure of cells to electromagnetic field results in changes in membrane potential. And the open probability of voltage sensitive calcium channel will be changed also. Thus the rate of $\mathrm{Ca}^{2+}$ and the pattern of calcium oscillations will be modulated. $v_{0}$ represents the influx rate of $\mathrm{Ca}^{2+}$ into the cell. The exposure of cell to electromagnetic field will modify $v_{0}$ as $v_{0} \times P_{0} / P_{0}^{\prime}$ in Eq. (1). $P_{0}^{\prime}$ is open probability of voltage sensitive calcium channel without electromagnetic field exposure, and $P_{0}$ is open probability during electromagnetic field exposure.

Because of the similarity for sodium and calcium ion channel topology, this work suppose that the voltage sensitive calcium channel's open probability is similar to the open probability of $\mathrm{N}_{\mathrm{a}}^{+}$channel [17]. 
Thus, we assume $P_{0}^{\prime}$ to be

$$
P_{0}^{\prime}=\frac{1}{1+e^{-\frac{z F\left(V_{0}-V_{H}\right)}{R T}}}
$$

in which $Z$ is the values for the equivalent number of gating particles, $V_{H}$ is the voltage at which the channel is open $50 \%$ of the time, $V_{0}$ is the initial equilibrium membrane potential, $T$ is the absolute temperature, $R$ is the universal gas constant, and $F$ is the Faraday constant.

According to Neves, et al. [18], $60 \mathrm{~Hz}$ electric field with low amplitudes can change the membrane potential in pancreatic $\beta$-cells. Then the work considers that the membrane potential is changed into $V_{0}+V_{m} \cos \omega t$ when the cell is exposed to extremely low frequency (ELF) electromagnetic fields. $V_{m}$ is the peak value of the $\mathrm{AC}$ voltage across the membrane, and $\omega$ is the angular frequency of the electromagnetic fields. The value of $V_{m}$ is given by the result of [19].

$$
V_{m}=\frac{\omega B_{0} c d}{2} \cdot \frac{\sigma_{i}}{\sigma_{m}}, \omega=2 \pi f
$$

Where $B_{0}$ represents magnetic density, $c$ represents the distance between cell and the center of electromagnetic field, $d$ represents the thickness of the plasma membrane. $\sigma_{i}$ and $\sigma_{m}$ represent conductivity of the intracellular fluid and membrane. In the following discussion, $P_{0}$ is written as

$$
P_{0}=\frac{1}{1+e^{-\frac{z F\left(V_{0}+V_{m} \cos \omega t-V_{H}\right)}{R T}}}
$$

$V_{0}$ is chosen as $-80 \mathrm{mV}, d$ is $20 \mathrm{~nm}, \sigma_{i} / \sigma_{m}=5 \times 10^{8}, z=3.7, c=5 \mathrm{~cm}$ and $V_{H}=-67.5 \mathrm{mV}$, see [17] and [19] for more details.

\section{Results and discussion}

Based on Eq. (5), some meaningful results have been obtained. Figure 1 shows the relationship between the voltage sensitive calcium channel's open probability and electromagnetic field intensity when the electromagnetic frequency is $50 \mathrm{~Hz}$. The results show that the open probability of voltage sensitive calcium channel increases with the enhancements of electromagnetic field intensity at first. Then $P_{0}$ keeps in about $50 \%$ when electromagnetic field intensity $\left(B_{0}\right)$ is greater than $4 \mathrm{mT}$.

In Figure 2, we present $P_{o}$ as a function of electromagnetic frequency $f$. One can see from Figure 2 that, $P_{o}$ keeps in peak when the electromagnetic frequency $f$ is $15 \mathrm{~Hz}$ and $60 \mathrm{~Hz}$. And it is in trough when the electromagnetic frequency $f$ is $35 \mathrm{~Hz}$ and $85 \mathrm{~Hz}$. This means that we would observe obvious biological effect when we select appropriate electromagnetic field frequency during the 


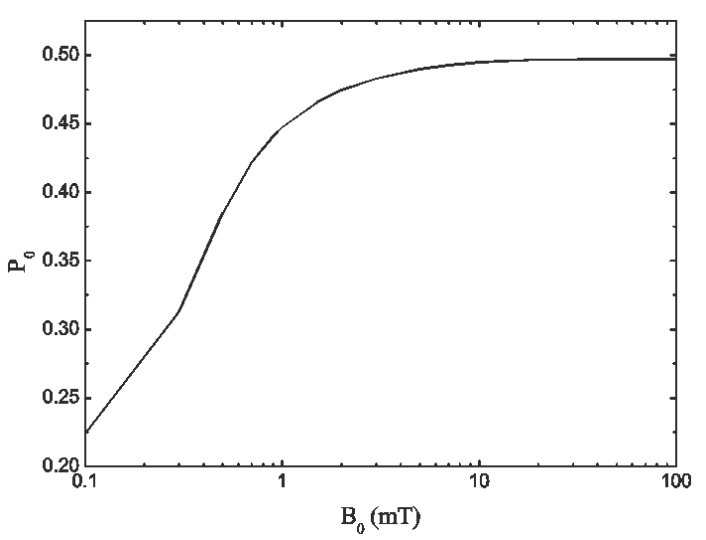

Fig. 1. The relationship between voltage sensitive calcium channel's open probability and electromagnetic field intensity.

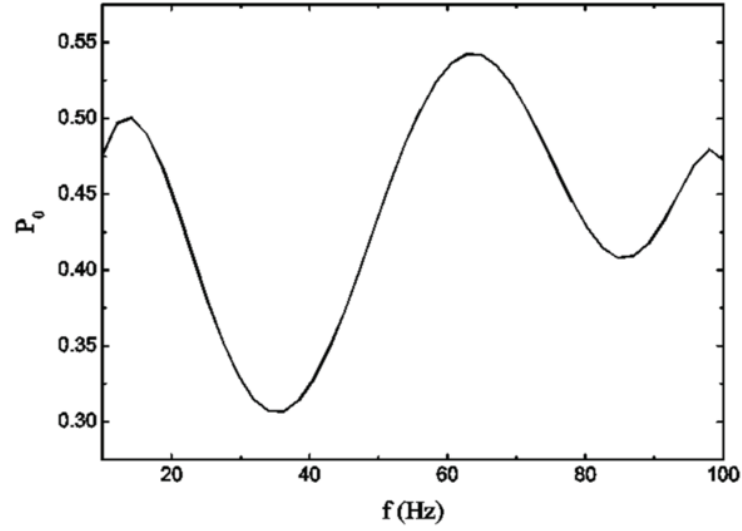

Fig. 2. The relationship between the voltage sensitive calcium channel's open probability and electromagnetic frequency.

research of biological effect of electromagnetic field.

The effects of noise on the calcium oscillations in a cell exposed to electromagnetic fields are discussed here. The curves of intracellular calcium concentration are presented in Figures 3(A) and 3(B) when the amplitude of electromagnetic fields $B_{0}$ is $10 m T$. In Figures 3(A) and 3(B), the curves of calcium oscillations are given when electromagnetic field frequencies are 50 and $100 \mathrm{~Hz}$, respectively. It can be well observed that the pattern of calcium oscillations changes with the increasing noise intensity.

Figure 4 shows that calcium oscillations cannot appear when there are no effects of noise on the cell (i.e. $D=0)$ under conditions that $B_{0}=0.01 \mathrm{mT}$ and $f=50 \mathrm{~Hz}$. And calcium oscillations can be induced clearly with the increasing noise intensity. This can be explained by the fact that the calcium oscillations could be induced by noise when the cell is exposed to some types of electromagnetic fields.

\section{Conclusion}
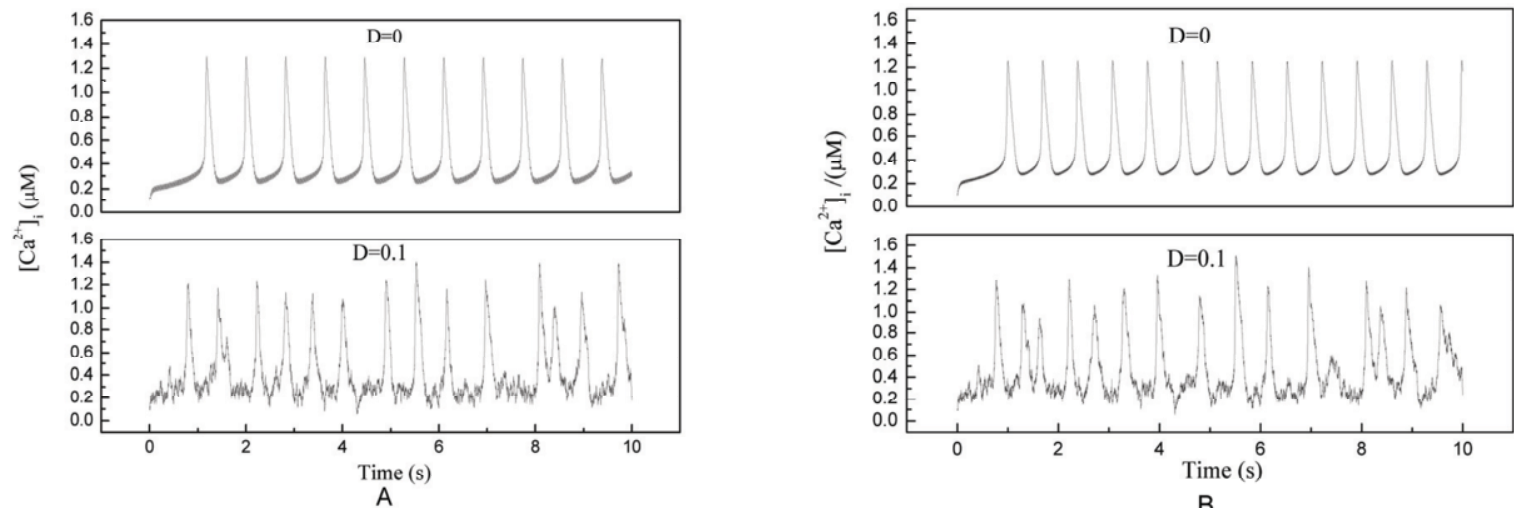

Fig. 3. Time evolution of the intracellular calcium concentration with different noise intensity. (A) $f=50 \mathrm{~Hz}$; (B) $f=100 \mathrm{~Hz}$ 

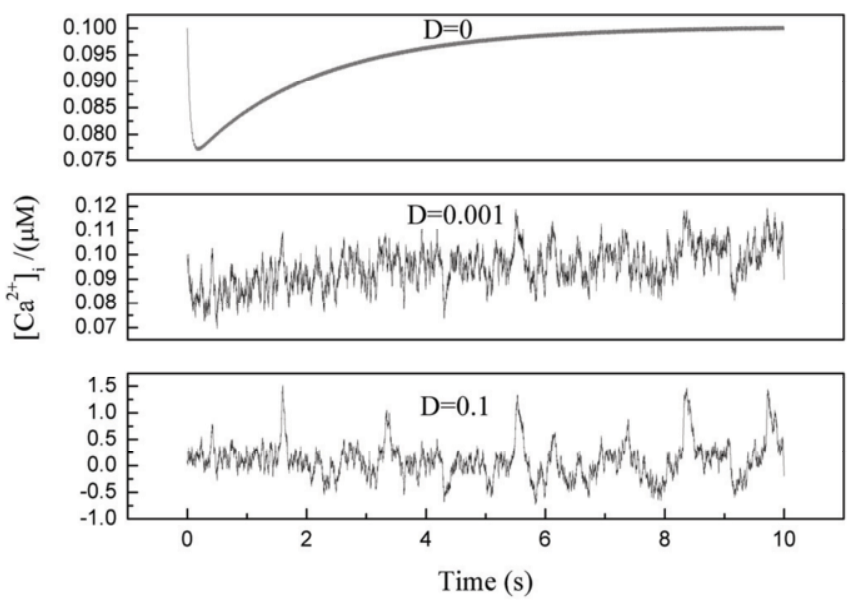

Fig. 4. Time evolution of the intracellular calcium concentration with different noise intensity. $\left(B_{0}=0.01 \mathrm{mT}, f=50 \mathrm{~Hz}\right)$.

In this paper, the effects of noise on the calcium oscillations in a cell exposed to electromagnetic fields were discussed. Noise is a very important factor to be considered in the dynamic research of the calcium oscillations in a cell exposed to electromagnetic fields. The work has shown that the pattern of intracellular calcium oscillations exposed to electromagnetic fields can be influenced as well as induced by noise. And the work has also studied the relationships between the open probability of voltage sensitive calcium channel and electromagnetic field. The result provides new insights into constructive roles and potential applications of selecting appropriate electromagnetic frequency during the research of biological effect of electromagnetic field.

\section{Acknowledgments}

The project is supported by the National Natural Science Foundation of China (11175055) and the Hebei Provincial Natural Science Foundation (A2015202268).

\section{References}

[1] M.J. Berridge, M.D. Bootman and P. Lipp, Calcium-a life and death signal, Nature 395 (1998), 645-648.

[2] R.E. Dolmetsch, K. Xu and R.S. Lewis, Calcium oscillations increase the efficiency and specificity of gene expression, Nature 392 (1998), 933-936.

[3] R.P. Liburdy, Calcium signaling in lymphocytes and ELF field evidence for an electric field metric and a site of interaction involving the calcium ion channel, FEBS Letters 301 (1992), 53-59.

[4] E. Lindstrom, P. Lindstrom, A. Berglund, K.H. Mild and E. Lundgren, Intracellular calcium oscillations induced in a Tcell line by a weak $50 \mathrm{~Hz}$ magnetic field, Journal of Cellular Physiology 156 (1993), 395-398.

[5] E. Lindstrom, P. Lindstrom, A. Berglund, E. Lundgren and K.H. Mild, Intracellular calcium oscillations in a T-cell line after exposure to extremely-low-frequency magnetic fields with variable frequencies and flux densities, Bioelectromagnetics 16 (1995), 41-47.

[6] X. Zhang, X.L. Liu, L T. Pan and I. Lee, Magnetic fields at extremely low-frequency $(50 \mathrm{~Hz}, 0.8 \mathrm{mT})$ can induce the uptake of intracellular calcium levels in osteoblasts, Biochemical and Biophysical Research Communications 396 (2010), $662-666$.

[7] C. Eichwald and F. Kaiser, Model for external influences on cellular signal transduction pathways including cytosolic calcium oscillations, Bioelectromagnetics 16 (1995), 75-85. 
[8] C.M. Huang, B.Q. Xu and J.R. Lin, Effects of extremely low frequency magnetic fields on hormone-induced cytosolic calcium oscillations, Acta Biochimica et Biophysica Sinica 15 (1999), 543-546.

[9] H. Zhang, J.D. Xu and Z.Q. Niu, The mechanism analysis on the intercellular calcium oscillations modulated by ELF magnetic fields, Medical Physics 24 (2007), 189-192.

[10] Y.H. Zhang, Y. Zhan, T.J. Zhao, Y.F. Chen and C.Q. Yuan, Model for influences of magnetic fields on intracellular calcium oscillations, Communications in Theoretical Physics 52 (2009), 168-172.

[11] Y.H. Zhang, Y.L. Zhao, Y.R. Han, H. Liu and Y. Zhan, Effects of magnetic fields on the synchronization of calcium oscillations in coupled cells, Journal of Computational and Theoretical Nanoscience 8 (2011), 1963-1966.

[12] M. Falcke, Reading the patterns in living cells-the physics of $\mathrm{Ca}^{2+}$ signaling, Advances in physics 53 (2004), 255-440.

[13]A. Goldbeter, G. Dupont and M.J. Berridge, Minimal model for signal-induced $\mathrm{Ca}^{2+}$ oscillations and for their frequency encoding through protein phosphorylation, Proceedings of the National Academy of Sciences of the United States of America 87 (1990), 1461-1465.

[14]F.J. Isaacs, J. Hasty, C.R. Cantor and J.J. Collins, Prediction and measurement of an auto regulatory genetic module, Proceedings of the National Academy of Sciences of the United States of America 100 (2003), 7714-7719.

[15]C.D. Grassi, M. Ascenzo, A. Torsello, G. Martinotti, F. Wolf, A. Cittadini and G.B. Azzena, Effects of $50 \mathrm{~Hz}$ electromagnetic fields on voltage-gated $\mathrm{Ca}^{2+}$ channels and their role in modulation of neuroendocrine cell proliferation and death, Cell Calcium 35 (2004), 307-315.

[16]H. Ebrahimian, M. Firoozabadi and M. Janahmadi, Parametric modeling of nerve cell under the sinusoidal environmental $50 \mathrm{~Hz}$ extremely low frequency magnetic field, Life Science Journal 10 (2013), 820-827.

[17] A.M. Correa, B. Francisco and L. Ramon, Gating kinetics of batrachotoxin-modified $\mathrm{Na}^{+}$channels in the squid giant axon, Biophysical Journal 61 (1992), 1332-1352.

[18] G.F. Neves, J.R.F. Silva, R.B. Moraes, T.S. Fernandes, B.M. Tenorio and R.A. Nogueira, $60 \mathrm{~Hz}$ electric field changes the membrane potential during burst phase in pancreatic $\beta$ - cells: In silico analysis, Acta Biotheoretica 62 (2014), 133143.

[19] C.Z. Jiang, C.Q. Luo and R.Z. Pan, Induced electric field and transmembrane potential of spherical cell in axis symmetric ELF magnetic field, Journal of Tianjin University(Science and Technology) 37 (2004), 713-716. 